\title{
Positive periodic solution for higher-order $p$-Laplacian neutral singular Rayleigh equation with variable coefficient
}

\author{
Yun Xin ${ }^{1}$, Shaowen Yao $^{2}$ and Zhibo Cheng ${ }^{2,3 *}$
}

\section{"Correspondence:}

czbo@hpu.edu.cn

${ }^{2}$ School of Mathematics and Information Science, Henan

Polytechnic University, Jiaozuo, 454000, China

${ }^{3}$ Department of Mathematics, Sichuan University, Chengdu, 610064, China

Full list of author information is available at the end of the article

\begin{abstract}
In this paper, we consider the existence of a positive periodic solution for the following kind of high-order $p$-Laplacian neutral singular Rayleigh equation with variable coefficient:

$$
\left(\varphi_{p}(x(t)-c(t) x(t-\sigma))^{(n)}\right)^{(m)}+f\left(t, x^{\prime}(t)\right)+g(t, x(t))=e(t)
$$

Our proof is based on Mawhin's continuation theory.

MSC: $34 \mathrm{C} 25 ; 34 \mathrm{~K} 13 ; 34 \mathrm{~K} 40$

Keywords: periodic solution; high-order; neutral operator; variable coefficient; singularity
\end{abstract}

\section{Introduction}

In this paper, we consider the following high-order $p$-Laplacian neutral singular Rayleigh equation with variable coefficient:

$$
\left(\varphi_{p}(x(t)-c(t) x(t-\sigma))^{(n)}\right)^{(m)}+f\left(t, x^{\prime}(t)\right)+g(t, x(t))=e(t),
$$

where $p>1, \varphi_{p}(x)=|x|^{p-2} x$ for $x \neq 0$ and $\varphi_{p}(0)=0, c \in C^{n}(\mathbb{R}, \mathbb{R})$ and $c(t+T) \equiv c(t), f$ is a continuous function defined in $\mathbb{R}^{2}$ and periodic in $t$ with $f(t, \cdot)=f(t+T, \cdot)$ and $f(t, 0)=$ $0, g(t, x)=g_{0}(x)+g_{1}(t, x)$, where $g_{1}: \mathbb{R} \times(0,+\infty) \rightarrow \mathbb{R}$ is an $L^{2}$-Carathéodory function, $g_{1}(t, \cdot)=g_{1}(t+T, \cdot), g_{0} \in C((0, \infty) ; \mathbb{R})$ has a singularity at $x=0, e: \mathbb{R} \rightarrow \mathbb{R}$ is a continuous periodic function with $e(t+T) \equiv e(t)$ and $\int_{0}^{T} e(t) d t=0, T$ is a positive constant, and $n$ and $m$ are positive integers.

In recent years, there are many works on periodic solutions for high-order neutral differential equations (see [1-11] and the references therein). Wang and Lu [5] in 2007 investigated the existence of periodic solution for the following high-order neutral functional differential equation with distributed delay:

$$
(x(t)-c x(t-\sigma))^{(n)}+f(x(t)) x^{\prime}(t)+g\left(\int_{-r}^{0} x(t+s) d \alpha(s)\right)=p(t) .
$$

(c) The Author(s) 2017. This article is distributed under the terms of the Creative Commons Attribution 4.0 International License (http://creativecommons.org/licenses/by/4.0/), which permits unrestricted use, distribution, and reproduction in any medium, provided you give appropriate credit to the original author(s) and the source, provide a link to the Creative Commons license, and indicate if changes were made. 
Using the continuation theorem of coincidence degree theory, they obtained the existence of periodic solutions for (1.2). Afterwards, Ren et al. considered the following high-order $p$-Laplacian neutral differential equation

$$
\left(\varphi_{p}(x(t)-c x(t-\sigma))^{(l)}\right)^{(n-l)}=F\left(t, x(t), x^{\prime}(t), \ldots, x^{(l-1)}(t)\right) .
$$

They obtained the existence of periodic solutions for (1.3) in the general case $(|c| \neq 1)$ in [10] and in the critical case $(|c|=1)$ in [9], respectively.

At the same time, some authors began to consider high-order neutral differential equation with singularity. Recently, applying the coincidence degree theory and some analysis skills, Xin et al. [11] discussed the existence of a positive periodic solution for the following neutral Liénard equation with singularity:

$$
\left(\varphi_{p}(x(t)-c x(t-\tau))^{(n)}\right)^{(m)}+f(x(t)) x^{\prime}(t)+g(t, x(t-\sigma))=e(t) .
$$

Inspired by these results in [5,9-11], in this paper, we consider the existence of a positive periodic solution for (1.1) with singularity by applications of Mawhin's continuation theory. The obvious difficulty lies in the following two respects. Firstly, $(x(t)-c(t) x(t-\sigma))^{(n)} \neq$ $x^{(n)}(t)-c(t) x^{(n)}(t-\sigma)$, and the calculation of $(x(t)-c(t) x(t-\sigma))^{(n)}$ is very complicated. Secondly, a priori bounds of periodic solutions are not easy to estimate.

\section{Preparation}

Firstly, we give qualitative properties of the neutral operator $(A x)(t):=x(t)-c(t) x(t-\sigma)$.

Lemma 2.1 (see [12]) If $|c(t)| \neq 1$, then the operator $A$ has a continuous inverse $A^{-1}$ on $C_{T}:=\{\phi \in C(\mathbb{R}, \mathbb{R}): \phi(t+T) \equiv \phi(t)\}$, satisfying

$$
\left|\left(A^{-1} f\right)(t)\right| \leq \frac{|f|_{\infty}}{\Gamma}, \quad \forall f \in C_{T}
$$

where $\Gamma:= \begin{cases}1-|c| \infty & \text { for }|c| \infty:=\max _{t \in[0, T T}|c(t)|<1, \\ |c|_{0}-1 & \text { for }|c|_{0}:=\min _{t \in[0, T]}|c(t)|>1\end{cases}$

Lemma 2.2 (Gaines and Mawhin [13]) Let $X$ and $Y$ be two Banach spaces, and let L: $D(L) \subset X \rightarrow Y$ be a Fredholm operator with index zero. Let $\Omega \subset X$ be an open bounded set, and let $N: \bar{\Omega} \rightarrow Y$ be L-compact on $\bar{\Omega}$. Assume that the following conditions hold:

(1) $L x \neq \lambda N x, \forall x \in \partial \Omega \cap D(L), \lambda \in(0,1)$;

(2) $N x \notin \operatorname{Im} L, \forall x \in \partial \Omega \cap \operatorname{Ker} L$;

(3) $\operatorname{deg}\{J Q N, \Omega \cap \operatorname{Ker} L, 0\} \neq 0$, where $J: \operatorname{Im} Q \rightarrow \operatorname{Ker} L$ is an isomorphism.

Then the equation $L x=N x$ has a solution in $\bar{\Omega} \cap D(L)$.

Lemma 2.3 (see [11]) If $x \in C_{T}^{1}:=\left\{x \in C^{1}(\mathbb{R}, \mathbb{R}): x(t+T) \equiv x(t)\right\}$ and there exists a point $t_{0} \in(0, T)$ such that $\left|x\left(t_{0}\right)\right|<d$, then

$$
|x|_{\infty} \leq d+\frac{1}{2} \int_{0}^{T}\left|x^{\prime}(t)\right| d t
$$

where $|x|_{\infty}:=\max _{t \in \mathbb{R}}|x(t)|$. 
To use the continuation degree theorem, we rewrite (1.1) in the form

$$
\left\{\begin{array}{l}
\left(A x_{1}\right)^{(n)}(t)=\varphi_{q}\left(x_{2}(t)\right) \\
x_{2}^{(m)}(t)=-f\left(t, x_{1}^{\prime}(t)\right)+g\left(t, x_{1}(t)\right)+e(t)
\end{array}\right.
$$

where $\frac{1}{p}+\frac{1}{q}=1$. Clearly, if a periodic solution of (2.1) is $x(t):=\left(\begin{array}{l}x_{1} \\ x_{2}\end{array}\right)$, then $x_{1}(t)$ must be a periodic solution of (1.1). Thus, the problem of finding a periodic solution for (1.1) reduces to finding a periodic solution for (2.1).

Now, set

$$
X:=\left\{x \in C\left(\mathbb{R}, \mathbb{R}^{2}\right): x(t+T) \equiv x(t)\right\}
$$

with the norm $|x|_{\infty}=\max \left\{\left|x_{1}\right|_{\infty},\left|x_{2}\right|_{\infty}\right\}$ and

$$
Y:=\left\{x \in C^{1}\left(\mathbb{R}, \mathbb{R}^{2}\right): x(t+T) \equiv x(t)\right\}
$$

with the norm $\|x\|=\max \left\{|x|_{\infty},\left|x^{\prime}\right|_{\infty}\right\}$. Clearly, both $X$ and $Y$ are Banach spaces. Meanwhile, define

$$
L: D(L)=\left\{x \in C^{n+m}\left(\mathbb{R}, \mathbb{R}^{2}\right): x(t+T)=x(t), t \in \mathbb{R}\right\} \subset X \rightarrow Y
$$

by

$$
(L x)(t)=\left(\begin{array}{c}
\left(A x_{1}\right)^{(n)}(t) \\
x_{2}^{(m)}(t)
\end{array}\right)
$$

and $N: X \rightarrow Y$ by

$$
(N x)(t)=\left(\begin{array}{c}
\varphi_{q}\left(x_{2}(t)\right) \\
-f\left(t, x_{1}^{\prime}(t)\right)-g\left(t, x_{1}(t)\right)+e(t)
\end{array}\right) .
$$

Then (2.1) can be converted into the abstract equation $L x=N x$.

If $x=\left(\begin{array}{l}x_{1} \\ x_{2}\end{array}\right) \in \operatorname{Ker} L$, that is, $\left\{\begin{array}{l}\left(x_{1}(t)-c(t) x_{1}(t-\sigma)\right)^{(n)}=0, \\ x_{2}^{(m)}(t)=0,\end{array}\right.$ then we have

$$
\left\{\begin{array}{l}
x_{1}(t)-c(t) x_{1}(t-\sigma)=a_{n-1} t^{n-1}+a_{n-2} t^{n-2}+\cdots+a_{1} t+a_{0}, \\
x_{2}(t)=b_{m-1} t^{m-1}+b_{m-2} t^{m-2}+\cdots+b_{1} t+b_{0},
\end{array}\right.
$$

where $a_{0}, \ldots, a_{n-1}, b_{0}, \ldots, b_{m-1} \in \mathbb{R}$ are constant. From $x_{1}(t)-c(t) x_{1}(t-\sigma) \in C_{T}$ and $x_{2}(t) \in$ $C_{T}$ we have $a_{1}=\cdots=a_{n-1}=0$ and $b_{1}=b_{2}=\cdots=b_{m-1}=0$. Let $\phi(t) \neq 0$ be a solution of $x(t)-c(t) x(t-\sigma)=1$. Then $\operatorname{Ker} L=x=\left(\begin{array}{c}a \phi(t), a \in \mathbb{R} \\ b, b \in \mathbb{R}\end{array}\right)$. From the definition of $L$ we can easily see that

$$
\operatorname{Ker} L \cong \mathbb{R}^{2}, \quad \operatorname{Im} L=\left\{y \in Y: \int_{0}^{T}\left(\begin{array}{l}
y_{1}(s) \\
y_{2}(s)
\end{array}\right) d s=\left(\begin{array}{l}
0 \\
0
\end{array}\right)\right\} .
$$

So $L$ is a Fredholm operator with index zero. 
Next, we will consider $L$-compact $N$. Let $P: X \rightarrow \operatorname{Ker} L$ and $Q: Y \rightarrow \operatorname{Im} Q \subset \mathbb{R}^{2}$ be defined by

$$
P x=\left(\begin{array}{c}
\left(A x_{1}\right)(0) \\
x_{2}(0)
\end{array}\right) \text { and } Q y=\frac{1}{T} \int_{0}^{T}\left(\begin{array}{l}
y_{1}(s) \\
y_{2}(s)
\end{array}\right) d s .
$$

Then $\operatorname{Im} P=\operatorname{Ker} L$ and $\operatorname{Ker} Q=\operatorname{Im} L$. Denote $L_{P}=\left.L\right|_{D(L) \cap \operatorname{Ker} P}$ and $\operatorname{let} L_{P}^{-1}: \operatorname{Im} L \rightarrow D(L)$ be the inverse of $L_{P}$. Then

$$
\begin{aligned}
& {\left[L_{P}^{-1} y\right](t)=\left(\begin{array}{c}
\left(A^{-1} G y_{1}\right)(t) \\
\left(G y_{2}\right)(t)
\end{array}\right),} \\
& {\left[G y_{1}\right](t)=\sum_{i=1}^{n-1} \frac{1}{i !} a_{i} t^{i}+\frac{1}{(n-1) !} \int_{0}^{t}(t-s)^{n-1} y_{1}(s) d s,} \\
& {\left[G y_{2}\right](t)=\sum_{i=1}^{m-1} \frac{1}{i !} b_{i} t^{i}+\frac{1}{(m-1) !} \int_{0}^{t}(t-s)^{m-1} y_{2}(s) d s,}
\end{aligned}
$$

where $a_{i}:=\left(A x_{1}\right)^{(i)}(0)$ are defined as follows:

$$
E_{1} Z=C \text {, where } E_{1}=\left(\begin{array}{cccccc}
1 & 0 & 0 & \cdots & 0 & 0 \\
e_{1} & 1 & 0 & \cdots & 0 & 0 \\
e_{2} & e_{1} & 1 & \cdots & 0 & 0 \\
\cdots & & & & & \\
e_{n-3} & e_{n-4} & e_{n-5} & \cdots & 1 & 0 \\
e_{n-2} & e_{n-3} & e_{n-4} & \cdots & e_{1} & 0
\end{array}\right)_{(n-1) \times(n-1)}
$$

$Z=\left(a_{n-1}, a_{n-2} \cdots, a_{1}\right)^{\top}, C=\left(c_{1}, c_{2}, \ldots, c_{n-1}\right)^{\top}, c_{i}=-\frac{1}{i ! T} \int_{0}^{T}(T-s)^{i} y_{1}(s) d s$, and $e_{j}=\frac{T^{j}}{(j+1) !}, j=$ $1,2, \ldots, n-2$. Similarly, we can get $b_{1}:=x_{2}^{(i)}(0), i=1,2, \ldots, m-1$. Therefore, from $(2.2)$ and (2.3) we get that $N$ is $L$-compact on $\bar{\Omega}$.

\section{Periodic solutions for (1.1) with repulsive singularity}

For convenience, we list the following assumptions, which will further used repeatedly:

$\left(\mathrm{H}_{1}\right)$ There exists a positive constant $K$ such that $|f(t, u)| \leq K$ for $(t, u) \in \mathbb{R} \times \mathbb{R}$.

$\left(\mathrm{H}_{2}\right)$ There exist positive constants $\alpha$ and $\beta$ such that $|f(t, u)| \leq \alpha|u|^{p-1}+\beta$ for $(t, u) \in$ $\mathbb{R} \times \mathbb{R}$

$\left(\mathrm{H}_{3}\right) f(t, u) \geq 0$ for $(t, u) \in \mathbb{R} \times \mathbb{R}$;

$\left(\mathrm{H}_{4}\right)$ There exists a positive constant $D$ such that $g(t, x)>K$ for $x>D$.

$\left(\mathrm{H}_{5}\right)$ There exists a positive constant $D_{1}$ such that $g(t, x)>|e|_{\infty}$ for $x>D_{1}$.

$\left(\mathrm{H}_{6}\right)$ There exist positive constants $\gamma, \zeta$ such that

$$
g(t, x) \leq \gamma x^{p-1}+\zeta \quad \text { for all } x>0 .
$$

$\left(\mathrm{H}_{7}\right)$ (Repulsive singularity) $\int_{0}^{1} g_{0}(s) d s=-\infty$. 
Theorem 3.1 Assume that $\left(\mathrm{H}_{1}\right),\left(\mathrm{H}_{4}\right)$, and $\left(\mathrm{H}_{6}\right)-\left(\mathrm{H}_{7}\right)$ hold. Then (1.1) has at least one T-periodic solution if

$$
0<\frac{T^{2 p}}{2^{2 p-1}}\left(\frac{T}{2 \pi}\right)^{(n-2)(p-1)+(m-2)} \frac{\gamma}{\left(\Gamma-\frac{T}{2} \sum_{k=0}^{n-1} C_{n}^{k} c_{n-k}\left(\frac{T}{2 \pi}\right)^{n-1-k}\right)^{p-1}}<1,
$$

where $c_{n-k}:=\max _{t \in[0, \omega]}\left|c^{(n-k)}(t)\right|$.

Proof Consider the abstract equation

$$
L x=\lambda N x, \quad \lambda \in(0,1)
$$

Set $\Omega_{1}=\{x: L x=\lambda N x, \lambda \in(0,1)\}$. If $x(t)=\left(x_{1}(t), x_{2}(t)\right)^{\top} \in \Omega_{1}$, then

$$
\left\{\begin{array}{l}
\left(A x_{1}\right)^{(n)}(t)=\lambda \varphi_{q}\left(x_{2}(t)\right) \\
x_{2}^{(m)}(t)=-\lambda f\left(t, x_{1}^{\prime}(t)\right)-\lambda g\left(t, x_{1}(t)\right)+\lambda e(t)
\end{array}\right.
$$

Substituting $x_{2}(t)=\lambda^{1-p} \varphi_{p}\left[\left(A x_{1}\right)^{(n)}(t)\right]$ into the second equation of (3.1), we have

$$
\left(\varphi_{p}\left(A x_{1}\right)^{(n)}(t)\right)^{(m)}+\lambda^{p} f\left(t, x_{1}^{\prime}(t)\right)+\lambda^{p} g\left(t, x_{1}(t)\right)=\lambda^{p} e(t) .
$$

Integrating both sides of (3.2) from 0 to $T$, we have

$$
\int_{0}^{T}\left(f\left(t, x_{1}^{\prime}(t)\right)+g\left(t, x_{1}(t)\right)\right) d t=0 .
$$

From the mean value theorem, there exists a point $\xi \in(0, T)$ such that

$$
f\left(\xi, x_{1}^{\prime}(\xi)\right)+g\left(\xi, x_{1}(\xi)\right)=0
$$

Then by $\left(\mathrm{H}_{1}\right)$ we have

$$
g\left(\xi, x_{1}(\xi)\right)=\left|-f\left(\xi, x_{1}^{\prime}(\xi)\right)\right| \leq K,
$$

and in view of $\left(\mathrm{H}_{4}\right)$, we get that $x_{1}(\xi) \leq D$. Since $x_{1}(t)$ is periodic with period $T$ and $x_{1}(t)>0$ for $t \in[0, T]$. Then $0<x_{1}(\xi) \leq D$. Therefore, from Lemma 2.3 we can get

$$
\left|x_{1}\right|_{\infty} \leq D+\frac{1}{2} \int_{0}^{T}\left|x_{1}^{\prime}(s)\right| d s
$$

From (3.4) and the Wirtinger inequality (see [14], Lemma 2.4) we get

$$
\begin{aligned}
\left|x_{1}\right|_{\infty} & \leq D+\frac{1}{2} T^{\frac{1}{2}}\left(\int_{0}^{T}\left|x_{1}^{\prime}(s)\right|^{2} d s\right)^{\frac{1}{2}} \\
& \leq D+\frac{1}{2} T^{\frac{1}{2}}\left(\frac{T}{2 \pi}\right)^{n-1}\left(\int_{0}^{T}\left|x_{1}^{(n)}(s)\right|^{2} d s\right)^{\frac{1}{2}} \\
& \leq D+\frac{T}{2}\left(\frac{T}{2 \pi}\right)^{n-1}\left|x_{1}^{(n)}\right|_{\infty} .
\end{aligned}
$$


Since $x_{1}^{(i-1)}(0)=x_{1}^{(i-1)}(T), i=1,2 \ldots, n-1$, there exists a point $t_{i}^{*} \in[0, T]$ such that $x_{1}^{(i)}\left(t_{i}^{*}\right)=$ 0 . From the Hölder and Wirtinger inequalities, we can easily get

$$
\begin{aligned}
\left|x_{1}^{(i)}\right|_{\infty} & \leq \frac{1}{2} \int_{0}^{T}\left|x_{1}^{(i+1)}(t)\right| d t \\
& \leq \frac{T^{\frac{1}{2}}}{2}\left(\int_{0}^{T}\left|x_{1}^{(i+1)}(t)\right|^{2} d t\right)^{\frac{1}{2}} \\
& \leq \frac{T}{2}\left(\frac{T}{2 \pi}\right)^{(n-i-1)}\left|x_{1}^{(n)}\right|_{\infty} .
\end{aligned}
$$

On the other hand, since $\left(A x_{1}\right)(t)=x_{1}(t)-c(t) x_{1}(t-\sigma)$, we have

$$
\begin{aligned}
\left(A x_{1}(t)\right)^{(n)}= & \left(x_{1}(t)-c(t) x_{1}(t-\sigma)\right)^{(n)} \\
= & x_{1}^{(n)}(t)-\left(c^{(n)}(t) x_{1}(t-\sigma)+n c^{(n-1)}(t) x_{1}^{\prime}(t-\sigma)\right. \\
& \left.+\frac{n(n-1)}{2 !} c^{(n-2)} x_{1}^{\prime \prime}(t-\sigma)+\cdots+c(t) x_{1}^{(n)}(t-\sigma)\right) \\
= & x_{1}^{(n)}(t)-c(t) x_{1}^{(n)}(t-\sigma)-\left(c^{(n)}(t) x_{1}(t-\sigma)+n c^{(n-1)}(t) x_{1}^{\prime}(t-\sigma)\right. \\
& \left.+\frac{n(n-1)}{2 !} c^{(n-2)} x_{1}^{\prime \prime}(t-\sigma)+\cdots+n c^{\prime}(t) x_{1}^{(n-1)}(t-\sigma)\right) .
\end{aligned}
$$

So, we can get

$$
\begin{aligned}
A x_{1}^{(n)}(t)= & \left(A x_{1}(t)\right)^{(n)}+\left(c^{(n)}(t) x_{1}(t-\sigma)+n c^{(n-1)}(t) x_{1}^{\prime}(t-\sigma)\right. \\
& \left.+\frac{n(n-1)}{2 !} c^{(n-2)} x_{1}^{\prime \prime}(t-\sigma)+\cdots+n c^{\prime}(t) x_{1}^{(n-1)}(t-\sigma)\right) .
\end{aligned}
$$

Applying Lemma 2.2, (3.5), and (3.6), we have

$$
\begin{aligned}
\left|x_{1}^{(n)}\right|_{\infty}= & \max _{t \in[0, T]}\left|A^{-1} A x_{1}^{(n)}(t)\right| \\
\leq & \left(\max _{t \in[0, T]} \mid\left(A x_{1}\right)^{(n)}(t)+c^{(n)}(t) x_{1}(t-\sigma)\right. \\
& \left.+n c^{(n-1)}(t) x_{1}^{\prime}(t-\sigma)+\cdots+n c^{\prime}(t) x_{1}^{(n-1)}(t-\sigma) \mid\right) / \Gamma \\
\leq & \frac{\varphi_{q}\left(\left|x_{2}\right|_{\infty}\right)+c_{n}\left|x_{1}\right|_{\infty}+n c_{n-1}\left|x_{1}^{\prime}\right|_{\infty}+\cdots+n c_{1}\left|x_{1}^{(n-1)}\right|_{\infty}}{\Gamma} \\
\leq & \left(\varphi_{q}\left(\left|x_{2}\right|_{\infty}\right)+c_{n}\left(D+\frac{T}{2}\left(\frac{T}{2 \pi}\right)^{n-1}\left|x_{1}^{(n)}\right|_{\infty}\right)\right. \\
& \left.+n c_{n-1}\left(\frac{1}{2} T\left(\frac{T}{2 \pi}\right)^{n-2}\left|x_{1}^{(n)}\right|_{\infty}\right)+\cdots+n c_{1} \frac{T}{2}\left|x_{1}^{(n)}\right|_{\infty}\right) / \Gamma
\end{aligned}
$$




$$
\begin{aligned}
\leq & \left(\varphi_{q}\left(\left|x_{2}\right|_{\infty}\right)+\frac{T}{2}\left(\left(\frac{T}{2 \pi}\right)^{n-1} c_{n}+n c_{n-1}\left(\frac{T}{2 \pi}\right)^{n-2}\right.\right. \\
& \left.\left.+\frac{n(n-1)}{2 !} c_{n-2}\left(\frac{T}{2 \pi}\right)^{n-3}+\cdots+n c_{1}\right)\left|x_{1}^{(n)}\right|_{\infty}+c_{n} D\right) / \Gamma \\
\leq & \frac{\varphi_{q}\left(\left|x_{2}\right|_{\infty}\right)+\frac{T}{2}\left(\sum_{k=0}^{n-1} C_{n}^{k} c_{n-k}\left(\frac{T}{2 \pi}\right)^{n-1-k}\right)\left|x_{1}^{(n)}\right|_{\infty}+c_{n} D}{\Gamma} .
\end{aligned}
$$

Since $\Gamma-\frac{T}{2}\left(\sum_{k=0}^{n-1} C_{n}^{k} c_{n-k}\left(\frac{T}{2 \pi}\right)^{n-1-k}\right)>0$, we have

$$
\left|x_{1}^{(n)}\right|_{\infty} \leq \frac{\varphi_{q}\left(\left|x_{2}\right|_{\infty}\right)+c_{n} D}{\Gamma-\frac{T}{2}\left(\sum_{k=0}^{n-1} C_{n}^{k} c_{n-k}\left(\frac{T}{2 \pi}\right)^{n-1-k}\right)} .
$$

In view of $\int_{0}^{T}\left(\varphi_{q}\left(x_{2}(t)\right)\right) d t=\int_{0}^{T}\left(A x_{1}(t)\right)^{(n)}(t) d t=0$, there exists a point $t_{2} \in(0, T)$ such that $x_{2}\left(t_{2}\right)=0$. From the Wirtinger inequality and from (3.4) we easily get

$$
\begin{aligned}
\left|x_{2}\right|_{\infty} & \leq \frac{1}{2} \int_{0}^{T}\left|x_{2}^{\prime}(t)\right| d t \leq \frac{\sqrt{T}}{2}\left(\int_{0}^{T}\left|x_{2}^{\prime}(t)\right|^{2}\right)^{\frac{1}{2}} \\
& \leq \frac{\sqrt{T}}{2}\left(\frac{T}{2 \pi}\right)^{m-2}\left(\int_{0}^{T}\left|x_{2}^{(m-1)}(t)\right|^{2} d t\right)^{\frac{1}{2}} \\
& \leq \frac{T}{2}\left(\frac{T}{2 \pi}\right)^{m-2}\left|x_{2}^{(m-1)}\right|_{\infty} .
\end{aligned}
$$

Besides, from $x_{2}^{(m-2)}(0)=x_{2}^{(m-2)}(T)$, there exists a point $t_{3} \in(0, T)$ such that $x_{2}^{(m-1)}\left(t_{3}\right)=0$, which, together with the integration of the second equation of (3.1) on interval $[0, T]$, yield

$$
\begin{aligned}
2\left|x_{2}^{(m-1)}(t)\right| & \leq 2\left(x_{2}^{(m-1)}\left(t_{3}\right)+\frac{1}{2} \int_{0}^{T}\left|x_{2}^{(m)}(t)\right| d t\right) \\
& \leq \lambda \int_{0}^{T}\left|f\left(t, x_{1}^{\prime}(t)\right)-g\left(t, x_{1}(t)\right)+e(t)\right| d t \\
& \leq \int_{0}^{T}\left|f\left(t, x_{1}^{\prime}(t)\right)\right| d t+\int_{0}^{T}|g(t, x(t))| d t+\int_{0}^{T}|e(t)| d t \\
& \leq K T+\int_{0}^{T}\left|g\left(t, x_{1}(t)\right)\right| d t+T|e|_{\infty},
\end{aligned}
$$

since $|f(t, u)| \leq K$ form $\left(\mathrm{H}_{1}\right)$. From $\left(\mathrm{H}_{1}\right)$ and $\left(\mathrm{H}_{6}\right)$ we have

$$
\begin{aligned}
\int_{0}^{T}\left|g\left(t, x_{1}(x)\right)\right| d t & =\int_{g\left(t, x_{1}(t)\right) \geq 0} g\left(t, x_{1}(t)\right) d t-\int_{g\left(t, x_{1}(t)\right) \leq 0} g\left(t, x_{1}(t)\right) d t \\
& =2 \int_{g\left(t, x_{1}(t)\right) \geq 0} g\left(t, x_{1}(t)\right) d t+\int_{0}^{T} f\left(t, x_{1}^{\prime}(t) d t\right. \\
& \leq 2 \int_{0}^{T}\left(\gamma x_{1}^{p-1}+\zeta\right) d t+\int_{0}^{T}\left|f\left(t, x_{1}^{\prime}(t)\right)\right| d t \\
& \leq 2 \gamma\left|x_{1}\right|_{\infty}^{p-1} T+2 \zeta T+K T .
\end{aligned}
$$


Since $(1+x)^{k} \leq 1+(1+k) x$ for $x \in[0, \delta]$, where $\delta$ is a constant, which depends only on $k>0$, substituting (3.10) into (3.9), we have

$$
\begin{aligned}
2\left|x_{2}^{(m-1)}(t)\right| & \leq 2 T \gamma\left|x_{1}\right|_{\infty}^{p-1}+2 \zeta T+2 K T+T|e|_{\infty} \\
& \leq 2 T \gamma\left(D+\frac{1}{2} \int_{0}^{T}\left|x_{1}^{\prime}(t)\right| d t\right)^{p-1}+N_{1} \\
& =2 T \gamma\left(1+\frac{D}{\frac{1}{2} \int_{0}^{T}\left|x_{1}^{\prime}(t)\right| d t}\right)^{p-1}\left(\frac{1}{2}\right)^{p-1}\left(\int_{0}^{T}\left|x_{1}^{\prime}(t)\right| d t\right)^{p-1}+N_{1} \\
& \leq \frac{1}{2^{p-2}} T \gamma\left(1+\frac{2 D p}{\int_{0}^{T}\left|x_{1}^{\prime}(t)\right| d t}\right)\left(\int_{0}^{T}\left|x_{1}^{\prime}(t)\right| d t\right)^{p-1}+N_{1},
\end{aligned}
$$

where $N_{1}:=2 \zeta T+2 K T+T|e|_{\infty}$. Substituting (3.6) and (3.7) into (3.11), we have

$$
\begin{aligned}
2\left|x_{2}^{(m-1)}(t)\right| & \leq \frac{T^{p} \gamma}{2^{p-2}}\left|x_{1}^{\prime}\right|_{\infty}^{p-1}+\frac{D p T^{p-1} \gamma}{2^{p-3}}\left|x_{1}^{\prime}\right|_{\infty}^{p-2}+N_{1} \\
\leq & \frac{T^{p} \gamma}{2^{p-2}} \cdot\left(\frac{T}{2}\right)^{p-1}\left(\frac{T}{2 \pi}\right)^{(n-2)) p-1)}\left|x_{1}^{(n)}\right|_{\infty}^{p-1} \\
& +\frac{D p T^{p-1} \gamma}{2^{p-3}}\left(\frac{T}{2}\right)^{p-2}\left(\frac{T}{2 \pi}\right)^{(n-2)(p-2)}\left|x_{1}^{(n)}\right|_{\infty}^{p-2}+N_{1} \\
\leq & \frac{T^{2 p-1} \gamma}{2^{2 p-3}}\left(\frac{T}{2 \pi}\right)^{(n-2)(p-1)} \frac{\left(\varphi_{q}\left(\left|x_{2}\right|_{\infty}\right)+C_{n} D\right)^{p-1}}{\left(\Gamma-\frac{T}{2}\left(\sum_{k=0}^{n-1} C_{n}^{k} c_{n-k}\left(\frac{T}{2 \pi}\right)^{n-1-k}\right)\right)^{p-1}} \\
& +\frac{D p T^{2 p-3} \gamma}{2^{2 p-5}}\left(\frac{T}{2 \pi}\right)^{(n-2)(p-2)} \frac{\left(\varphi_{q}\left(\left|x_{2}\right|_{\infty}\right)+C_{n} D\right)^{p-2}}{\left(\Gamma-\frac{T}{2}\left(\sum_{k=0}^{n-1} C_{n}^{k} c_{n-k}\left(\frac{T}{2 \pi}\right)^{n-1-k}\right)\right)^{p-2}}+N_{1} .
\end{aligned}
$$

Combining of (3.8) and (3.12) implies

$$
\begin{aligned}
\left|x_{2}\right|_{\infty} \leq & \frac{T}{2}\left(\frac{T}{2 \pi}\right)^{m-2}\left|x_{2}^{(m-1)}\right|_{\infty} \\
\leq & \frac{T}{4}\left(\frac{T}{2 \pi}\right)^{m-2}\left[\frac{T^{2 p-1} \gamma}{2^{2 p-3}}\left(\frac{T}{2 \pi}\right)^{(n-2)(p-1)} \frac{\left(\varphi_{q}\left(\left|x_{2}\right|_{\infty}\right)+C_{n} D\right)^{p-1}}{\left(\Gamma-\frac{T}{2}\left(\sum_{k=0}^{n-1} C_{n}^{k} c_{n-k}\left(\frac{T}{2 \pi}\right)^{n-1-k}\right)\right)^{p-1}}\right. \\
& \left.+\frac{D p T^{2 p-3} \gamma}{2^{2 p-5}}\left(\frac{T}{2 \pi}\right)^{(n-2)(p-2)} \frac{\left(\varphi_{q}\left(\left|x_{2}\right|_{\infty}\right)+C_{n} D\right)^{p-2}}{\left(\Gamma-\frac{T}{2}\left(\sum_{k=0}^{n-1} C_{n}^{k} c_{n-k}\left(\frac{T}{2 \pi}\right)^{n-1-k}\right)\right)^{p-2}}+N_{1}\right] .
\end{aligned}
$$

So, we have

$$
\begin{aligned}
\left|x_{2}\right|_{\infty} \leq & \frac{T^{2 p} \gamma}{2^{2 p-1}}\left(\frac{T}{2 \pi}\right)^{(n-2)(p-1)+(m-2)} \frac{\left|x_{2}\right|_{\infty}}{\left(\Gamma-\frac{T}{2}\left(\sum_{k=0}^{n-1} C_{n}^{k} c_{n-k}\left(\frac{T}{2 \pi}\right)^{n-1-k}\right)\right)^{p-1}} \\
& +\frac{T^{2 p} \gamma}{2^{2 p-1}}\left(\frac{T}{2 \pi}\right)^{(n-2)(p-1)+(m-2)} \frac{\left(\sum_{i=0}^{p-1} C_{p-1}^{i}\left(\left|x_{2}\right|_{\infty}^{q-1}\right)^{p-1-i}\left(c_{n} D\right)^{i}\right)}{\left(\Gamma-\frac{T}{2}\left(\sum_{k=0}^{n-1} C_{n}^{k} c_{n-k}\left(\frac{T}{2 \pi}\right)^{n-1-k}\right)\right)^{p-1}}
\end{aligned}
$$




$$
\begin{aligned}
& +\frac{D p T^{2 p-2} \gamma}{2^{2 p-3}}\left(\frac{T}{2 \pi}\right)^{(n-2)(p-2)+(m-2)} \frac{\left(\sum_{i=0}^{p-1} C_{p-2}^{i}\left(\left|x_{2}\right|_{\infty}^{q-1}\right)^{p-2-i}\left(c_{n} D\right)^{i}\right)}{\left(\Gamma-\frac{T}{2}\left(\sum_{k=0}^{n-1} C_{n}^{k} c_{n-k}\left(\frac{T}{2 \pi}\right)^{n-1-k}\right)\right)^{p-2}} \\
& +\frac{T}{4}\left(\frac{T}{2 \pi}\right)^{m-2} N_{1} .
\end{aligned}
$$

Since

$$
\frac{T^{2 p}}{2^{2 p-1}}\left(\frac{T}{2 \pi}\right)^{(n-2)(p-1)+(m-2)} \frac{\gamma}{\left(\Gamma-\frac{T}{2} \sum_{k=0}^{n-1} C_{n}^{k} c_{n-k}\left(\frac{T}{2 \pi}\right)^{n-1-k}\right)^{p-1}}<1,
$$

there exists a positive constant $M_{1}$ such that

$$
\left|x_{2}\right|_{\infty} \leq M_{1}
$$

Therefore, from (3.7) we have

$$
\begin{aligned}
\left|x_{1}^{(n)}\right|_{\infty} & \leq \frac{\varphi_{q}\left(\left|x_{2}\right|_{\infty}\right)+c_{n} D}{\Gamma-\frac{T}{2}\left(\sum_{k=0}^{n-1} C_{n}^{k} c_{n-k}\left(\frac{T}{2 \pi}\right)^{n-1-k}\right)} \\
& \leq \frac{M_{1}^{q-1}+c_{n} D}{\Gamma-\frac{T}{2} \sum_{k=0}^{n-1} C_{n}^{k} c_{n-k}\left(\frac{T}{2 \pi}\right)^{n-1-k}}:=M_{n}^{\prime} .
\end{aligned}
$$

From (3.6) we have

$$
\left|x_{1}^{\prime}\right|_{\infty} \leq \frac{T}{2}\left(\frac{T}{2 \pi}\right)^{n-2}\left|x_{1}^{(n)}\right|_{\infty} \leq \frac{T}{2}\left(\frac{T}{2 \pi}\right)^{n-2} M_{n}^{\prime}:=M_{2} .
$$

Hence, from (3.4) we have

$$
\left|x_{1}\right|_{\infty} \leq D+\frac{1}{2} \int_{0}^{T}\left|x_{1}^{\prime}(t)\right| d t \leq D+\frac{T M_{2}}{2}:=M_{3} .
$$

From (3.6), (3.9), and (3.10) we have

$$
\begin{aligned}
\left|x_{2}^{(m-1)}\right|_{\infty} & \leq \frac{1}{2} \max \left|\int_{0}^{T} x_{2}^{(m)}(t) d t\right| \\
& \leq \frac{1}{2} \int_{0}^{T}\left|-f\left(t, x_{1}^{\prime}(t)\right)-g\left(t, x_{1}(t)\right)+e(t)\right| d t \\
& \leq \frac{1}{2} \int_{0}^{T} \mid f\left(t, x_{1}^{\prime}(t)\left|d t+\frac{1}{2} \int_{0}^{T}\right| g\left(t, x_{1}(t)\left|d t+\frac{1}{2} \int_{0}^{T}\right| e(t) \mid d t\right.\right. \\
& \leq K T+m M_{3}^{p-1} T+n T+\frac{1}{2}|e|_{\infty} T:=M_{m-1} .
\end{aligned}
$$

From (3.8) we get

$$
\left|x_{2}^{\prime}\right|_{\infty} \leq \frac{T}{2}\left(\frac{T}{2 \pi}\right)^{m-3}\left|x_{2}^{(m-1)}\right|_{\infty} \leq \frac{T}{2}\left(\frac{T}{2 \pi}\right)^{m-3} M_{m-1}:=M_{4} .
$$

On the other hand, since $g\left(t, x_{1}\right)=g_{1}\left(t, x_{1}(t)\right)+g_{0}\left(x_{1}(t)\right)$, (3.2) can be rewritten as

$$
\left(\varphi_{p}\left(A x_{1}\right)^{(n)}\right)^{(m)}+\lambda^{p} f\left(t, x_{1}^{\prime}(t)\right)+\lambda^{p}\left(g_{1}\left(t, x_{1}(t)\right)+g_{0}(x(t))\right)=\lambda^{p} e(t) .
$$


Let $\tau \in[0, T]$ for any $\tau \leq t \leq T$. Multiplying both sides of (3.19) by $x_{1}^{\prime}(t)$ and integrating on $[\tau, t]$, we have

$$
\begin{aligned}
\lambda^{p} \int_{x_{1}(\tau)}^{x_{1}(t)} g_{0}(u) d u= & \lambda^{p} \int_{\tau}^{t} g_{0}\left(x_{1}(s)\right) x_{1}^{\prime}(s) d s \\
= & -\int_{\tau}^{t}\left(\varphi_{p}\left(A x_{1}\right)^{(n)}(s)\right)^{(m)} x_{1}^{\prime}(s) d s-\lambda^{p} \int_{\tau}^{t} f\left(s, x_{1}^{\prime}(s)\right) x_{1}^{\prime}(s) d s \\
& -\lambda^{p} \int_{\tau}^{t} g_{1}\left(s, x_{1}(s)\right) x_{1}^{\prime}(s) d s+\lambda^{p} \int_{\tau}^{t} e(s) x_{1}^{\prime}(s) d s .
\end{aligned}
$$

By (3.2), (3.12), (3.17), and (3.18) we have

$$
\begin{aligned}
& \left|\int_{\tau}^{t}\left(\varphi_{p}\left(A x_{1}\right)^{(n)}(s)\right)^{(m)} x_{1}(s) d s\right| \\
& \quad \leq \int_{\tau}^{t}\left|\left(\varphi_{p}\left(A x_{1}\right)^{(n)}(s)\right)^{(m)}\right|\left|x_{1}^{\prime}(s)\right| d s \\
& \quad \leq\left|x_{1}^{\prime}\right|_{\infty} \int_{\tau}^{t}\left|\left(\varphi_{p}\left(A x_{1}\right)^{(n)}(s)\right)^{(m)}\right| d s \\
& \quad \leq \lambda^{p} M_{2}\left(\int_{0}^{T}\left|f\left(t, x_{1}^{\prime}(t)\right)\right| d t+\int_{0}^{T}\left|g\left(t, x_{1}(t)\right)\right| d t+\int_{0}^{T}|e(t)| d t\right) \\
& \quad \leq \lambda^{p} M_{2}\left(2 K T+2 m M_{3}^{p-1} T+2 n T+|e|_{\infty} T\right) .
\end{aligned}
$$

Moreover, we have

$$
\begin{aligned}
& \left|\int_{\tau}^{t} f\left(s, x_{1}^{\prime}(s)\right) x_{1}^{\prime}(s) d s\right| \leq\left|x_{1}^{\prime}\right|_{\infty} \int_{0}^{T}\left|f\left(t, x_{1}^{\prime}(t)\right)\right| d t \leq M_{2} K T \\
& \left|\int_{\tau}^{t} g_{1}\left(s, x_{1}(s)\right) x_{1}^{\prime}(s) d s\right| \leq\left|x_{1}^{\prime}\right|_{\infty} \int_{0}^{T}\left|g_{1}\left(t, x_{1}(t)\right)\right| d t \leq M_{2} \sqrt{T}\left\|g_{M_{3}}\right\|_{2} \\
& \left|\int_{\tau}^{t} e(s) x_{1}^{\prime}(s) d s\right| \leq\left|x_{1}^{\prime}\right|_{\infty} \int_{0}^{T}|e(t)| d t \leq M_{2}|e|_{\infty} T
\end{aligned}
$$

where $g_{M_{3}}:=\max _{0<x \leq M_{3}}\left|g_{1}(t, u)\right| \in L^{2}(0, T)$ and $\left\|g_{M_{3}}\right\|_{2}:=\left(\int_{0}^{T}\left|g_{1}\left(t, x_{1}^{\prime}(t)\right)\right|^{2} d t\right)^{\frac{1}{2}}$. Substituting (3.21) and (3.22) into (3.20), we have

$$
\left|\int_{x_{1}(\tau)}^{x_{1}(t)} g_{0}(x) d x\right| \leq M_{2}\left(3 K T+2 m M_{3}^{p-1} T+2 n T+\sqrt{T}\left\|g_{M_{3}}\right\|_{2}+2|e|_{\infty} T\right):=M_{5}^{*} .
$$

From repulsive singular condition $\left(\mathrm{H}_{7}\right)$ we know that there exists a constant $M_{5}>0$ such that

$$
x_{1}(t) \geq M_{5}, \quad \forall t \in[\tau, T] .
$$

The case $t \in[0, \tau]$ can be treated similarly.

Let

$$
\Omega_{2}=\left\{x=\left(x_{1}, x_{2}\right)^{\top}: E_{5}<x_{1}(t)<E_{1},\left|x_{1}^{\prime}\right|_{\infty}<E_{2},\left|x_{2}\right|_{\infty}<E_{3},\left|x_{2}^{\prime}\right|_{\infty}<E_{4}\right\}
$$


where $0<E_{5}<M_{5}, E_{1}>\max \left\{D, M_{3}\right\}, E_{2}>M_{2}, E_{3}>M_{1}$, and $E_{4}>M_{4}$. Next, we shall prove that $\Omega_{2}$ is a bounded set. In fact, for all $x \in \Omega_{2}, x_{2}=0, x_{1}=a_{0} \phi(t)$, and $a_{0} \in \mathbb{R}^{+}$, we have

$$
0=\int_{0}^{T} g\left(t, a_{0} \phi(t)\right) d t
$$

From assumption $\left(\mathrm{H}_{1}\right)$ we have $0<a_{0} \phi(t) \leq D$. So $\Omega_{2}$ is a bounded set.

Let $\Omega=\left\{x \in\left(x_{1}, x_{2}\right)^{\top}:\|x\| \leq M\right\}$, where $M=\max \left\{E_{1}, E_{2}, E_{3}, E_{4}\right\}$. Then $\Omega_{1} \cup \Omega_{2} \subset \Omega$, and, as it follows from the above proof, $L x \neq \lambda N x$ for all $(x, \lambda) \in \partial \Omega \times(0,1)$, so that conditions (1) and (2) of Lemma 2.2 are both satisfied. Define the isomorphism $J: \operatorname{Im} Q \rightarrow \operatorname{Ker} L$ as follows:

$$
J\left(x_{1}, x_{2}\right)^{\top}=\left(x_{2},-x_{1}\right)^{\top}
$$

Let $H(\mu, x)=-\mu x+(1-\mu) J Q N x,(\mu, x) \in[0,1] \times \Omega$. Then, for all $(\mu, x) \in(0,1) \times(\partial \Omega \cap$ $\operatorname{Ker} L)$,

$$
H(\mu, x)=\left(\begin{array}{c}
-\mu x_{1}(t)-\frac{1-\mu}{T} \int_{0}^{T} g\left(t, x_{1}(t)\right) d t \\
-\mu x_{2}(t)-(1-\mu) \varphi_{q}\left(x_{2}(t)\right)
\end{array}\right)
$$

since $\int_{0}^{T} e(t) d t=0$ and $f(t, 0)=0$. From $\left(\mathrm{H}_{4}\right)$ it is obvious that $x^{\top} H(\mu, x)<0$ for all $(\mu, x) \in$ $(0,1) \times(\partial \Omega \cap \operatorname{Ker} L)$. Hence

$$
\begin{aligned}
\operatorname{deg}\{J Q N, \Omega \cap \operatorname{Ker} L, 0\} & =\operatorname{deg}\{H(0, x), \Omega \cap \operatorname{Ker} L, 0\} \\
& =\operatorname{deg}\{H(1, x), \Omega \cap \operatorname{Ker} L, 0\} \\
& =\operatorname{deg}\{I, \Omega \cap \operatorname{Ker} L, 0\} \neq 0 .
\end{aligned}
$$

So condition (3) of Lemma 2.2 is satisfied. Applying Lemma 2.2, we conclude that equation $L x=N x$ has a solution $x=\left(x_{1}, x_{2}\right)^{\top}$ on $\bar{\Omega} \cap D(L)$, that is, (1.1) has a $T$-periodic solution $x_{1}(t)$.

Theorem 3.2 Assume that $\left(\mathrm{H}_{2}\right)-\left(\mathrm{H}_{3}\right)$ and $\left(\mathrm{H}_{5}\right)-\left(\mathrm{H}_{7}\right)$ hold. Then (1.1) has at least a nonconstant T-periodic solution if

$$
0<\frac{\left(\frac{T^{2 p} \gamma}{2^{2 p-1}}+\frac{T^{p+1} \alpha}{2^{p}}\right)\left(\frac{T}{2 \pi}\right)^{m+n-4}}{\left(\Gamma-\frac{T}{2} \sum_{k=0}^{n-1} C_{n}^{k} c_{n-k}\left(\frac{T}{2 \pi}\right)^{n-1-k}\right)^{p-1}}<1 .
$$

Proof We follow the same strategy and notation as the proof of Theorem 3.1. Now, we consider $\left\|x^{\prime}\right\| \leq M_{2}$.

We first claim that there is a constant $\xi^{*} \in[0, T]$ such that

$$
0<x_{1}\left(\xi^{*}\right) \leq D_{1}
$$

Since $\int_{0}^{T}\left(\varphi_{p}\left(A x_{1}\right)^{\prime}(t)\right)^{\prime} d t=0$, there exist two points $\xi^{*}, \xi_{*} \in[0, T]$ such that

$$
\left(\varphi_{p}\left(A x_{1}\right)^{\prime}\left(\xi^{*}\right)\right)^{\prime} \geq 0 \quad \text { and } \quad\left(\varphi_{p}\left(A x_{1}\right)^{\prime}\left(\xi_{*}\right)\right)^{\prime} \leq 0 .
$$


From $\left(\mathrm{H}_{3}\right)$ and (3.2) we have

$$
g\left(\xi^{*}, x_{1}\left(\xi^{*}\right)\right)-e\left(\xi^{*}\right) \leq-f\left(\xi^{*}, x_{1}^{\prime}\left(\xi^{*}\right)\right) \leq 0
$$

since $f\left(\xi^{*}, x_{1}^{\prime}\left(\xi^{*}\right)\right)>0$. Therefore, we get

$$
g\left(\xi^{*}, x_{1}^{\prime}\left(\xi^{*}\right)\right) \leq e\left(\xi^{*}\right) \leq|e|_{\infty}
$$

From $\left(\mathrm{H}_{5}\right)$ we have

$$
x_{1}(\xi) \leq D_{1}
$$

Since $x(t)>0$, we get $0<x_{1}\left(\xi^{*}\right) \leq D_{1}$. This proves (3.24).

Similarly, from (3.4) we have

$$
\left|x_{1}(t)\right| \leq D_{1}+\frac{1}{2} \int_{0}^{T}\left|x_{1}^{\prime}(t)\right| d t .
$$

From (3.9) and $\left(\mathrm{H}_{2}\right)$ we get

$$
\begin{aligned}
2\left|x_{2}^{(m-1)}(t)\right| & \leq 2\left(x_{2}^{(m-1)}\left(t_{3}\right)+\frac{1}{2} \int_{0}^{T}\left|x_{2}^{(m)}(t)\right| d t\right) \\
& \leq \lambda \int_{0}^{T}\left|f\left(t, x_{1}^{\prime}(t)\right)-g\left(t, x_{1}(t)\right)+e(t)\right| d t \\
& \leq \int_{0}^{T}\left|f\left(t, x_{1}^{\prime}(t)\right)\right| d t+\int_{0}^{T}|g(t, x(t))| d t+\int_{0}^{T}|e(t)| d t \\
& \leq \alpha \int_{0}^{T}\left|x_{1}^{\prime}(t)\right|^{p-1} d t+\beta T+\int_{0}^{T}\left|g\left(t, x_{1}(t)\right)\right| d t+T|e|_{\infty} .
\end{aligned}
$$

From (3.10), $\left(\mathrm{H}_{2}\right)$, and $\left(\mathrm{H}_{7}\right)$ we have

$$
\begin{aligned}
\int_{0}^{T}\left|g\left(t, x_{1}(t)\right)\right| d t & =\int_{g\left(t, x_{1}(t)\right) \geq 0} g\left(t, x_{1}(t)\right) d t-\int_{g\left(t, x_{1}(t)\right)<0} g\left(t, x_{1}(t)\right) d t \\
& =2 \int_{g\left(t, x_{1}(t)\right) \geq 0} g\left(t, x_{1}(t)\right) d t+\int_{0}^{T} f\left(t, x_{1}^{\prime}(t)\right) d t \\
& \leq 2 \int_{g\left(t, x_{1}(t)\right) \geq 0}\left(\gamma x_{1}^{p-1}(t)+\zeta\right) d t+\int_{0}^{T}\left|f\left(t, x_{1}^{\prime}(t)\right)\right| d t \\
& \leq 2 \gamma\left|x_{1}\right|^{p-1} T+2 \zeta T+\alpha \int_{0}^{T}\left|x_{1}^{\prime}(t)\right|^{p-1} d t+\beta T .
\end{aligned}
$$

Substituting (3.27) into (3.26), from (3.11) we have

$$
\begin{aligned}
2\left|x_{2}^{(m-1)}(t)\right| & \leq 2 \gamma \int_{0}^{T}|x(t)|^{p-1} d t+2 \zeta T+2 \alpha \int_{0}^{T}\left|x^{\prime}(t)\right|^{p-1} d t+2 \beta T+|e|_{\infty} T \\
& \leq\left(\frac{T^{p} \gamma}{2^{p-2}}+2 \alpha T\right)\left|x_{1}^{\prime}\right|_{\infty}^{p-1}+\frac{D p T^{p-1} \gamma}{2^{p-3}}\left|x_{1}^{\prime}\right|_{\infty}^{p-2}+N_{2},
\end{aligned}
$$


where $N_{2}=2 T(\zeta+\beta)+\|e\| T$. From (3.12), (3.13), and (3.14) we get

$$
\begin{aligned}
\left|x_{2}\right|_{\infty} \leq & \left(\frac{T^{2 p} \gamma}{2^{2 p-1}}+\frac{T^{p+1} \alpha}{2^{p}}\right)\left(\frac{T}{2 \pi}\right)^{(n-2)(p-1)+(m-2)} \frac{\left|x_{2}\right|_{\infty}}{\left(\Gamma-\frac{T}{2}\left(\sum_{k=0}^{n-1} C_{n}^{k} c_{n-k}\left(\frac{T}{2 \pi}\right)^{n-1-k}\right)\right)^{p-1}} \\
& +\frac{T^{2 p} \gamma}{2^{2 p-1}}\left(\frac{T}{2 \pi}\right)^{(n-2)(p-1)+(m-2)} \frac{\left(\sum_{i=0}^{p-1} C_{p-1}^{i}\left(\left|x_{2}\right|_{\infty}^{q-1}\right)^{p-1-i}\left(c_{n} D\right)^{i}\right)}{\left(\Gamma-\frac{T}{2}\left(\sum_{k=0}^{n-1} C_{n}^{k} c_{n-k}\left(\frac{T}{2 \pi}\right)^{n-1-k}\right)\right)^{p-1}} \\
& +\frac{D p T^{2 p-2} \gamma}{2^{2 p-3}}\left(\frac{T}{2 \pi}\right)^{(n-2)(p-2)+(m-2)} \frac{\left(\sum_{i=0}^{p-1} C_{p-2}^{i}\left(\left|x_{2}\right|_{\infty}^{q-1}\right)^{p-2-i}\left(c_{n} D\right)^{i}\right)}{\left(\Gamma-\frac{T}{2}\left(\sum_{k=0}^{n-1} C_{n}^{k} c_{n-k}\left(\frac{T}{2 \pi}\right)^{n-1-k}\right)\right)^{p-2}} \\
& +\frac{T}{4}\left(\frac{T}{2 \pi}\right)^{m-2} N_{2} .
\end{aligned}
$$

Since $\frac{\left(\frac{T^{2} p_{\gamma}}{2^{2 p-1}}+\frac{T^{p+1}{ }^{p}}{2^{p}}\right)\left(\frac{T}{2 \pi}\right)^{(n-2)(p-1)+(m-2)}}{\left(\Gamma-\frac{T}{2} \sum_{k=0}^{n-1} C_{n}^{k} c_{n-k}\left(\frac{T}{2 \pi}\right)^{n-1-k}\right)^{p-1}}<1$, it is easy to see that there exists a positive constant $M_{2}$ such that

$$
\left\|x^{\prime}\right\| \leq M_{2}
$$

The rest of the proof is the same as in Theorem 3.1.

We illustrate our results with an example.

Example 3.1 Consider the neutral functional differential

$$
\begin{aligned}
& \left(\varphi_{p}\left(x(t)-\frac{1}{64} \sin (4 t) x(t-\sigma)\right)^{\prime \prime \prime}\right)^{\prime \prime \prime}+\cos ^{2}(2 t) \sin x^{\prime}(t)+\frac{1}{4 \pi}(\sin (4 t)+3) x^{3}(t)-\frac{1}{x^{\mu}} \\
& \quad=5 \cos (4 t)
\end{aligned}
$$

where $p=4, \sigma$ and $\mu$ are constants, and $0<\sigma<T$. It is clear that $T=\frac{\pi}{2}, n=3, m=3$, $c(t)=\frac{1}{64} \sin 4 t, e(t)=5 \cos 4 t, c_{1}=\max _{t \in[0, T]}\left|\frac{1}{16} \cos 4 t\right|=\frac{1}{16}, c_{2}=\max _{t \in[0, T]}\left|-\frac{1}{4} \sin 4 t\right|=\frac{1}{4}$, and $c_{3}=\max _{t \in[0, T]}|-\cos 4 t|=1$. In this case, $f(t, u)=\cos ^{2}(2 t) \sin u, f(t, 0)=0,|f(t, u)|=$ $\left|\cos ^{2}(2 t) \sin u\right| \leq 1, K=1$; and $g(t, x)=\frac{1}{4 \pi}(\sin 4 t+3) x^{3}(t)-\frac{1}{x^{\mu}} \leq \frac{1}{\pi} x^{3}+1, \gamma=\frac{1}{\pi}, \zeta=1$; Obviously, conditions $\left(\mathrm{H}_{1}\right)$ and $\left(\mathrm{H}_{6}\right)-\left(\mathrm{H}_{7}\right)$ hold. Choose $D=4 \pi$ such that $\left(\mathrm{H}_{4}\right)$ holds. Now we consider the following condition:

$$
\begin{aligned}
& \frac{T^{2 p}}{2^{2 p-1}}\left(\frac{T}{2 \pi}\right)^{(n-2)(p-1)+(m-2)} \frac{\gamma}{\left(\Gamma-\frac{T}{2} \sum_{k=0}^{n-1} C_{n}^{k} c_{n-k}\left(\frac{T}{2 \pi}\right)^{n-1-k}\right)^{p-1}} \\
& \quad=\frac{\left(\frac{\pi}{2}\right)^{8}}{2^{7}}\left(\frac{\frac{\pi}{2}}{2 \pi}\right)^{4} \frac{\frac{1}{\pi}}{\left(\frac{63}{64}-\frac{\pi}{4} \times\left(1 \times \frac{1}{16}+3 \times \frac{1}{4} \times \frac{1}{4}+3 \times \frac{1}{16}\right)\right)^{3}} \\
& \approx \frac{\pi^{8}}{2^{26}}<1 .
\end{aligned}
$$

So, by Theorem 3.1, (3.29) has at least one nonconstant $\frac{\pi}{2}$-periodic solution.

\section{Conclusions}

In summary, a periodic solution of (1.1) with singularity is illustrated by Theorems 3.1 and 3.2. In Theorem 3.1, we consider the existence of a periodic solution for (1.1) in the case 
$|f(t, u)| \leq K$. Furthermore, in Theorem 3.2, we give a condition on $f(t, u)$ that is weaker than $|f(, u)| \leq K$ in Theorem 3.1, that is, we obtain the existence of periodic solution for (1.1) in the case where $|f(t, u)| \leq \alpha|u|^{p-1}+\beta$. From the mathematical point of view, the results are valuable to understand the periodic solutions for high-order neutral differential equations.

\section{Acknowledgements}

YX, SWY, and ZBC would like to thank the referee for invaluable comments and insightful suggestions. This work was supported by National Natural Science Foundation of China (No. 11501170), China Postdoctoral Science Foundation funded project (2016M590886), Education Department of Henan Province project (No. 16B1 10006), Henan Polytechnic University Outstanding Youth Fund (J2015-02), and Henan Polytechnic University Doctor Fund (B2013-055).

\section{Competing interests}

The authors declare that they have no competing interests.

\section{Authors' contributions}

$Y X, S W Y$, and ZBC worked together in the derivation of the mathematical results. All authors read and approved the final manuscript.

\section{Author details}

${ }^{1}$ College of Computer Science and Technology, Henan Polytechnic University, Jiaozuo, 454000, China. ${ }^{2}$ School of Mathematics and Information Science, Henan Polytechnic University, Jiaozuo, 454000, China. ${ }^{3}$ Department of Mathematics, Sichuan University, Chengdu, 610064, China.

\section{Publisher's Note}

Springer Nature remains neutral with regard to jurisdictional claims in published maps and institutional affiliations.

Received: 8 August 2017 Accepted: 6 October 2017 Published online: 19 October 2017

\section{References}

1. Bai, Z, Zhang, S, Sun, S, Yin, C: Monotone iterative method for a class of fractional differential equations. Electron. J. Differ. Equ. 2016, 6 (2016)

2. Cheng, Z, Ren, J: Periodic solution for high-order differential system. J. Appl. Anal. Comput. 3, 239-249 (2013)

3. Cong, F: Periodic solutions for $2 k$ th order ordinary differential equations with nonresonance. Nonlinear Anal., Theory Methods Appl. 32, 787-793 (1998)

4. Dong, X, Bai, Z, Zhang, S: Positive solutions to boundary value problems of $p$-Laplacian with fractional derivative. Bound. Value Probl. 2017, 5 (2017)

5. Wang, K, Lu, S: On the existence of periodic solutions for a kind of high-order neutral functional differential equation. J. Math. Anal. Appl. 326, 1161-1173 (2007)

6. Li, X, Lu, S: Periodic solution for a kind of high-order $p$-Laplacian differential equation with sign-changing coefficient ahead of non-linear term. Nonlinear Anal., Theory Methods Appl. 70, 1011-1022 (2009)

7. Li, Y: Existence and exponential stability of almost periodic solution for neutral delay BAM neural networks with time-varying delays in leakage terms. J. Franklin Inst. 350, 2808-2825 (2013)

8. Pan, L: Periodic solutions for higher order differential equations with deviating argument. J. Math. Anal. Appl. 343 904-918 (2008)

9. Ren, J, Cheng, Z: Periodic solutions for generalized high-order neutral differential equation in the critical case. Nonlinear Anal., Theory Methods Appl. 71, 6182-6193 (2009)

10. Ren, J, Cheung, W, Cheng, Z: Existence and Lyapunov stability of periodic solutions for generalized higher-order neutral differential equations. Bound. Value Probl. 2011, 1 (2011)

11. Xin, $Y$, Han, $X$, Cheng, Z: Positive periodic solution for high-order $p$-Laplacian neutral differential equation with singularity. Bound. Value Probl. 2016, 33 (2016)

12. Du, B, Guo, L, Ge, W, Lu, S: Periodic solutions for generalized Liénard neutral equation with variable parameter. Nonlinear Anal., Theory Methods Appl. 70, 2387-2394 (2009)

13. Gaines, R, Mawhin, J: Coincidence Degree and Nonlinear Differential Equation. Springer, Berlin (1977)

14. Torres, $P, C$ Cheng, $Z$, Ren, J: Non-degeneracy and uniqueness of periodic solutions for $2 n$-order differential equations. Discrete Contin. Dyn. Syst. 33, 2155-2168 (2013) 